\title{
PITPNC1 fuels radioresistance of rectal cancer by inhibiting reactive oxygen species production
}

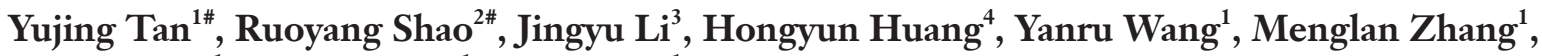 \\ Jianyun $\mathrm{Cao}^{1}$, Junde Zhang ${ }^{1}$, Junguo $\mathrm{Bu}^{1}$
}

${ }^{1}$ Department of Radiation Oncology, Zhujiang Hospital, Southern Medical University, Guangzhou 510280, China; ${ }^{2}$ Department of Hematology, Nanfang Hospital, Southern Medical University, Guangzhou 510515, China; ${ }^{3}$ Department of Pathology, ${ }^{4}$ Department of Abdominal Surgery, Zhujiang Hospital, Southern Medical University, Guangzhou 510280, China

Contributions: (I) Conception and design: Y Tan, J Zhang, J Bu; (II) Administrative support: J Zhang, J Bu; (III) Provision of study materials or patients: Y Tan, J Li, H Huang, M Zhang; (IV) Collection and assembly of data: Y Tan, J Li, H Huang, M Zhang; (V) Data analysis and interpretation: R Shao, Y Wang, J Cao; (VI) Manuscript writing: All authors; (VII) Final approval of manuscript: All authors.

\#These authors contributed equally to this work.

Correspondence to: Junde Zhang, MD; Junguo Bu, MD. Department of Radiation Oncology, Zhujiang Hospital, Southern Medical University, Guangzhou 510280, China. Email: 13002087575@163.com; flkd007@smu.edu.cn.

\begin{abstract}
Background: Neoadjuvant radiotherapy is a commonly used method for the current standard-of-care for most patients with rectal cancer, when the effects of radioresistance are limited. The phosphatidylinositol transfer protein, cytoplasmic 1 (PITPNC1), a lipid-metabolism-related gene, has previously been proved to manifest pro-cancer effects in multiple types of cancer. However, whether PITPNC1 plays a role for developing radioresistance in rectal cancer patients is still unknown. Therefore, this study aims to investigate the role of PITPNC1 in rectal cancer radioresistance.
\end{abstract}

Methods: Patient-derived tissue were used to detect the difference in the expression level of PITPNC1 between radioresistant and radiosensitive patients. Bioinformatic analyses of high-throughput gene expression data were applied to uncover the correlations between PITPNC1 level and oxidative stress. Two rectal cancer cell lines, SW620, and HCT116, were selected in vitro to investigate the effect of PITPNC1 on radioresistance, reactive oxygen species (ROS) generation, apoptosis, and proliferation in rectal cancer.

Results: PITPNC1 is highly expressed in radioresistant patient-derived rectal cancer tissues compared to radiosensitive tissue; therefore, PITPNC1 inhibits the generation of ROS and improves the extent of radioresistance of rectal cancer cell lines and then inhibits apoptosis. Knocking down PITPNC1 facilitates the production of ROS while application of the ROS scavenger, N-acetyl-L-cysteine (NAC), could reverse this effect.

Conclusions: PITPNC1 fuels radioresistance of rectal cancer via the inhibition of ROS generation.

Keywords: Rectal cancer; radioresistance; phosphatidylinositol transfer protein, cytoplasmic 1 (PITPNC1); reactive oxygen species generation (ROS generation); oxidative stress

Submitted Jan 05, 2020. Accepted for publication Feb 01, 2020.

doi: $10.21037 /$ atm.2020.02.37

View this article at: http://dx.doi.org/10.21037/atm.2020.02.37

\section{Introduction}

Colorectal cancer is the third most common cancer, and rectal cancer accounts for approximately $30 \%$ of the total cancer occurrences (1). Radiotherapy is an important method in neoadjuvant therapy for patients with rectal cancer, which improves the anus-preservation rate and lowers the risk of recurrence. Despite $22-30 \%$ of patients being able to achieve pathological complete remission after neoadjuvant radiotherapy, 30-50\% of them are 
unlikely to benefit significantly from it, and $5 \%$ may even suffer metastasis (2-6). The possibility of developing radioresistance hampers the effect of neoadjuvant radiotherapy and worsens clinical outcomes. Although there have already been many attempts to seek radiosensitivity biomarkers (7-12), less has been known about the exact mechanisms of the development of radioresistance in rectal cancer, which is vital to improve the therapeutic effect of rectal cancer further and to develop a reliable radiosensitivity evaluation method to optimize clinical decision-making.

It has been well proven that the main mechanisms of the therapeutic effects of radiotherapy to cancer is DNA double-strand break induced by reactive oxygen species (ROS). The source of more than $90 \%$ of total ROS generated is the oxidation respiratory chain located in the mitochondria (13). Previous studies have highlighted the importance of glycolysis-related metabolic reprogramming mechanisms of tumor cells in radioresistance development (14-17). However, lipid oxidative metabolism-related mechanisms were also recently demonstrated to play a negative regulatory role in this process $(18,19)$.

Phosphatidylinositol transfer protein, cytoplasmic 1 (PITPNC1), is a member of the phosphatidylinositol transfer proteins (PITPs) family that participates in multiple cellular processes, including lipid metabolism (20-24). PITPNC1, specifically, is characterized to bind and transfer phosphatidic acid, and thus is a key member in the process of phosphatidic acid metabolism (25). Previous studies have demonstrated that PITPNC1 plays a pro-cancer role by promoting tumor angiogenesis, metastasis, and malignant secretion (26,27). Our recent work have also shown that PITPNC1 fuels omental metastasis of gastric cancer (28). Nevertheless, whether PITPNC1 participates in the development of radioresistance is still unknown. This study aims to explore the effect of PITPNC1 on radioresistance in rectal cancer. We reveal here that PITPNC1 promotes radioresistance by inhibiting ROS production in rectal cancer.

\section{Methods}

\section{Patient population}

This study was approved by the Zhujiang Hospital (Guangzhou, China) Ethics Review Board. Frozen and formalin-fixed, paraffin-embedded rectal cancer tissue samples obtained from 16 patients were used in this study.
Samples were taken from patients who underwent surgical treatment in the Zhujiang Hospital (Guangzhou, China) without other pre-surgical intervention.

\section{Western blotting}

Western blotting was performed complying with the standards set by our previous study (28). Cells required irradiation continued to be cultured for $48 \mathrm{~h}$ after the exposure to irradiation (0 Gy for negative controls and 4 Gy for experiment groups). The total protein of tissues and cells were homogenized using lysis buffer containing protease inhibitors (keyGEN, Nanjing, China) on ice. After centrifugation, the supernatant containing protein was collected. Total protein and $5 \times$ SDS loading buffer were mixed and boiled at $100{ }^{\circ} \mathrm{C}$ for $5 \mathrm{~min}$. Samples were separated using electrophoresis on $10 \%$ SDSpolyacrylamide gel and then transferred onto polyvinylidene fluoride membranes. The membranes were subsequently blocked for $1 \mathrm{~h}$ at room temperature with $5 \%$ skim milk supplemented with $0.1 \%$ Tween 20 (TBST). Membranes were then incubated overnight with a primary antibody at $4{ }^{\circ} \mathrm{C}$ and then with a secondary antibody for $60 \mathrm{~min}$ at room temperature. Immunoreactive bands were then visualized using a chemiluminescence (ECL) detection system or LI-COR Odyssey infrared imaging system. All used first antibodies include: PITPNC1 (Sigma, Saint Louis, MO, USA), Cleaved Caspase-3 (Cell Signaling Technology, Danvers, MA, USA), $\gamma \mathrm{H} 2 \mathrm{AX}$ (Abcam, Cambridge, MA, USA), $\beta$-actin (Proteintech, Chicago, IL, USA).

\section{Histological analysis}

Immunohistochemical (IHC) staining was performed using patient-derived, formalin-fixed, and paraffin-embedded rectal cancer tissue samples. Samples were then sectioned and immunostained as previously reported (28). The primary antibody PITPNC1 (Sigma, Saint Louis, MO, USA, 1:200 dilution) was used. The PV-9000 general twostep test kit was used for secondary antibody incubation and staining.

\section{Cell culture and transfection}

Six human rectal cancer cell lines, including HCA7, DIFI, Coco-2, HCT116, SW620, and LOVO, were obtained from Foleibao Biotechnology Development Co. (Shanghai, China). All cell lines were cultured as previously reported (28) 
in RPMI 1640 medium supplemented with $10 \%$ fetal bovine serum (HyClone, Logan, UT, USA) in a $37{ }^{\circ} \mathrm{C}$ incubator under $5 \% \mathrm{CO}_{2}$. For the knocking down of PITPNC1, shPITPNC1 (sequence: CCGGCAATGGATGAAGTCC GAGAATCTCGAGATTCTCGGACTTCATCCATTGT TTTTG) was applied. For the clearance of ROS, the ROS scavenger, N-acetyl-L-cysteine (NAC), was employed, and $2.5 \mathrm{nM}$ NAC was added in each plate.

\section{Cell survival assay}

MTT assay was adopted to analyze the survival rate of cells. All cells were plated in 96-well plates (approximately 5,000 cells per well) with 5 replicates and exposed to irradiation of 4 Gy using a linear accelerator (Elekta, Sweden). Twenty $\mu \mathrm{L}$ 3-(4,5-Dimethylthiazol-2-Yl)-2,5-Diphenyltetrazolium Bromide (MTT) solution $(5 \mathrm{mg} / \mathrm{mL})$ was then added to each well. The original medium containing MTT was completely removed after incubated for $4 \mathrm{~h}$, after which $150 \mu \mathrm{L}$ dimethylsulfoxide (DMSO) was added to each well. The optical density (OD) was measured using a SpectraMax M5 microplate reader (Molecular Devices, Sunnyvale, CA, USA) at $490 \mathrm{~nm}$ on day $0,1,2,3$, and 4 . The cell survival rate of day $\mathrm{n}(\%)$ was calculated as $\left(\mathrm{OD}_{570}\right.$ of day $\mathrm{n} / \mathrm{OD}_{570}$ of day 0$) \times 100 \%$.

\section{Colony formation assay}

Cultured cells were exposed to irradiation (0 Gy for negative controls and 4 Gy for experiment groups) and then trypsinized and plated in 6-well plates at a density of 500 cells per well. The medium was replaced every second day. Cells were cultured under $5 \% \mathrm{CO}_{2}$ at $37{ }^{\circ} \mathrm{C}$ for 2 weeks. Cells were then fixed using $4 \%$ paraformaldehyde and stained with crystal violet. The numbers of colonies were counted under microscope.

\section{Flow cytometry assay}

Cells were seeded on 6 -well plates at a density of $2 \times 10^{5}$ cells per well. Forty-eight h after the exposure to irradiation (0 Gy for negative control and 4 Gy for experiment group), cells were trypsinized and collected for later experiments. For the analysis of apoptosis, Annexin V-FITC Apoptosis Detection Kit (KeyGEN, Nanjing, China) was applied according to the manufacturer's protocol. For the measurement of ROS production, fluorescent 2',7'-dichlorofluorescein diacetate (DCF-DA) was used, and ROS was detected as described in the manufacturer's protocol in the commercial kit (Nanjing Jiancheng, China).

\section{Bioinformatic analyses}

The microarray data of all cases with radiosensitivity data in GSE56699 was downloaded under the GPL14951 platform (Illumina HumanHT-12 WG-DASL V4.0 R2 expression beadchip) from the Gene Expression Omnibus (GEO). The RNA-seq data from a total of 166 rectal cancer cases were downloaded from The Cancer Genome Atlas (TCGA) database. For the microarray data, the R package 'limma' was applied for differential gene analysis. For the RNA-seq data, the R package 'EdgeR' was used for differential gene analysis. All bioinformatics analyses using the $\mathrm{R}$ language was performed with R 3.5.3 (R Project for Statistical Computing, Vienna, Austria). Gene ontology (GO) and Kyoto Encyclopedia of Genes and Genomes (KEGG) enrichment analyses were performed using the online tool Database for Annotation, Visualization, and Integrated Discovery (DAVID) v6.8. Gene Set Enrichment Analysis (GSEA) was performed using the software GSEA v4.0.3 (29,30).

\section{Statistical analyses}

Each experiment was performed in at least triplicate. All statistical analyses were performed using the SPSS 22 software (SPSS, Chicago, IL, USA). Differences between experimental groups were assessed by Student's t-test (two groups) or one-way analysis of variance (ANOVA) (more than two groups). All values were expressed as mean $\pm \mathrm{SD}$, and statistical significance was defined as $\mathrm{P}<0.05$. *, $\mathrm{P}<0.05$; **, $\mathrm{P}<0.01$; ***, $\mathrm{P}<0.001 ;$ ****, $\mathrm{P}<0.0001$.

\section{Results}

\section{The relevance of the high expression of PITPNC1 with radioresistance in rectal cancer tissues}

To investigate the relevance of PITPNC1 expression level and radioresistance in rectal cancer, we first performed a differential analysis using MicroArray data of 11 rectal cancer cases ( 5 radioresistant vs. 6 radiosensitive) from GSE56699. Compared to patients that did not manifest radioresistance, patients that did have significantly higher level of PITPNC1 expression (Figure 1A). To further validate this finding, the PITPNC1 protein level was detected in a total of 16 patient-derived rectal cancer 

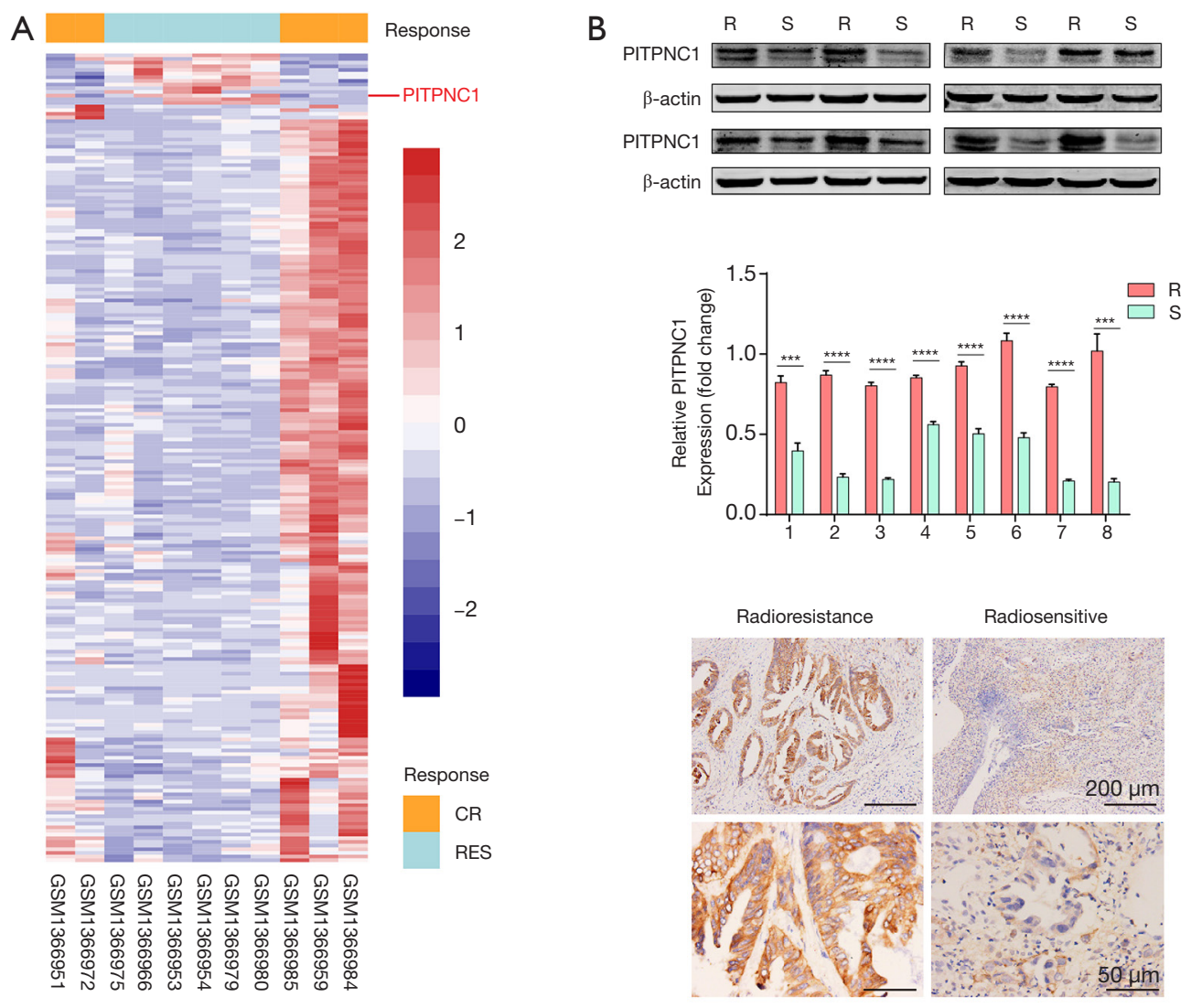

Figure 1 High expression of PITPNC1 in radioresistant rectal cancer tissue. (A) Heatmap of Illumina HumanHT-12 WG-DASL V4.0 R2 expression beadchip data of rectal cancer tissue derived from 11 patients in GSE56699; (B) western blotting analysis of PITPNC1 level in colorectal cancer tissue from patients who manifested radioresistance and those who did not. B-actin was used as a loading control; (C) quantization of western blotting result using relative expression level of PITPNC1 compared to $\beta$-actin; (D) immunohistochemistry results in the comparison of the expression level of PITPNC1 in radioresistant colorectal cancer tissue and radiosensitive tissue. Representative photographs of the two groups are shown. R, resistant; S, sensitive; RES, resistant; CR, complete remission; PITPNC1, phosphatidylinositol transfer protein, cytoplasmic 1 .

specimens from our center classified as radioresistant (R) or radiosensitive (S) according to medical record using western blotting (Figure $1 B, C$ ) and immunohistochemistry staining (Figure 1D), and similar results were obtained. These results taken together show that the expression level of PITPNC1 is significantly higher in radioresistant rectal cancer tissues compared to radiosensitive tissues.

\section{High PITPNC1 expression in rectal cancer is associated with radioresistance and less ROS production}

MTT assay was performed using common rectal cancer cell lines, including HCA7, DIF1, Coco-2, HCT116, SW620, and LOVO cultured under 4 Gy irradiation. Results showed that HCT116 had the strongest radioresistance, while SW620 had the weakest radioresistance (Figure 2A). These two cell lines were selected as representative radioresistant and radiosensitive rectal cancer cell lines for the following experiments. The expression level of PITPNC1 in HCT116 was significantly higher than SW620, as shown by western blotting (Figure 2B,C). We next investigated whether the correlation between high PITPNC1 expression and radioresistance was mediated by reduced ROS generation. Flow cytometry was adopted to assess the ROS production level of irradiated and non-irradiated SW620 and HCT116 cells. We observed a significantly higher ROS generation level in SW620 compared to HCT116 in both irradiated and non-irradiated groups (Figure 2D,E). In order 
A

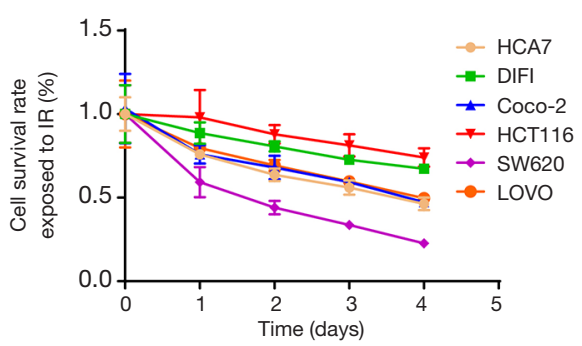

D
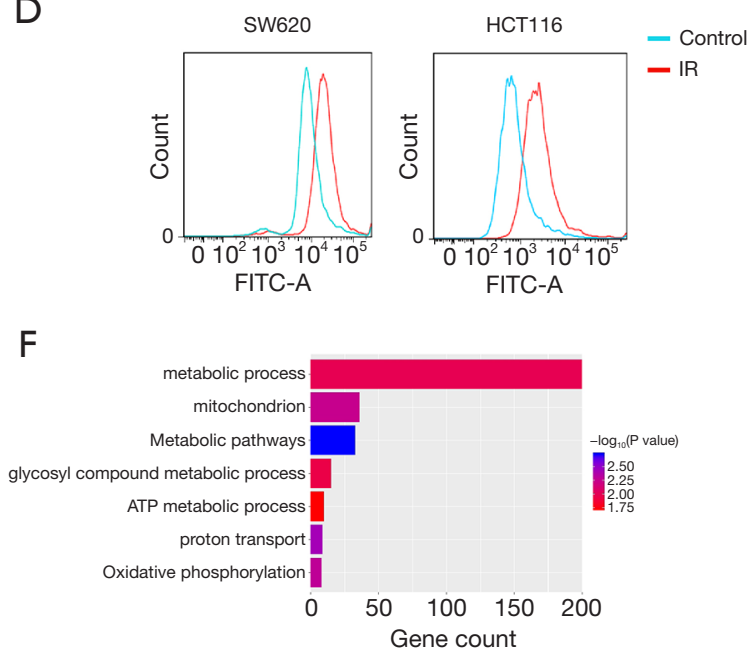

$\mathrm{B}$ P

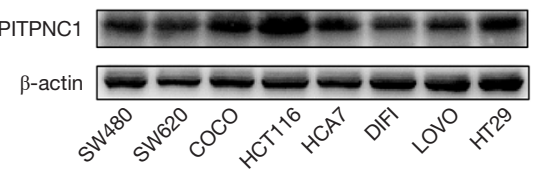

C

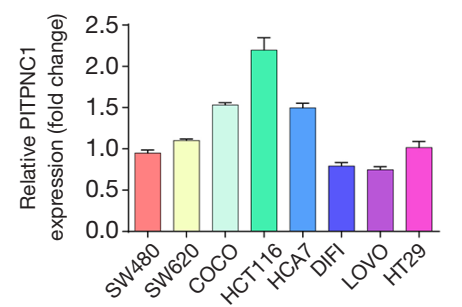

$E$

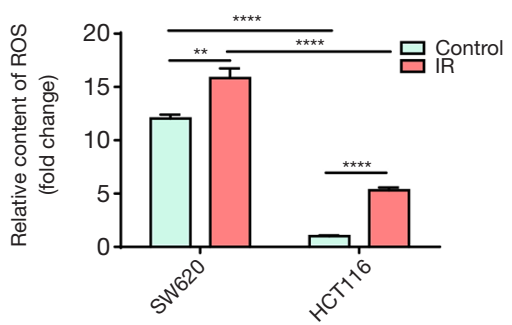

G

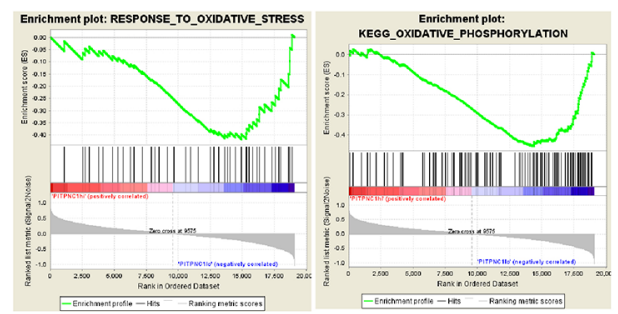

Figure 2 High PITPNC1 expression in rectal cancer is associated with radioresistance and decreased ROS production. (A) MTT assay results of rectal cancer cell lines including HCA7, DIF1, Coco-2, HCT116, SW620 and LOVO exposed under irradiation; (B) western Blot analysis of PITPNC1 in rectal cancer cell lines with $\beta$-actin as the loading control; (C) quantization of western blotting result using relative expression level of PITPNC1 compared to $\beta$-actin; (D) flow cytometry results of ROS production in irradiated cells (red) compared to non-treated controls (blue) in SW620 and HCT116 cell lines; (E) quantization of flow cytometry results showing the relative content of ROS in SW620 and HCT116 cell lines using fold change normalized by controls; (F) GO \& KEGG Enrichment analysis of TCGA rectum adenocarcinoma RNAseq data using down-regulated genes in cases with high PITPNC1 level compared with those with low PITPNC1 level; (G) GSEA results of the cases with the 25\% highest PITPNC1 level compared to those with 25\% lowest PITPNC1 level derived TCGA rectum adenocarcinoma RNAseq data on oxidative phosphorylation and oxidative stress. IR, irradiation; PITPNC1, phosphatidylinositol transfer protein, cytoplasmic 1; ROS, reactive oxygen species; GO, gene ontology; KEGG, Kyoto Encyclopedia of Genes and Genomes; GSEA, Gene Set Enrichment Analysis; TCGA, The Cancer Genome Atlas. ${ }^{* *}, \mathrm{P}<0.01$; ****, $\mathrm{P}<0.0001$.

to comprehensively analyze the difference in oxidative stress condition between rectal cancer tissue with high PITPNC1 level and low PITPNC1 level, we obtained RNA-seq data of 166 rectal cancer cases from TCGA. Cases were ranked according to the PITPNC1 level, and the 42 cases $(25 \%$, respectively) with the highest and the lowest PITPNC1 level were classified as "PITPNC1 hi" and "PITPNC1 lo". The differential analysis identified 347 upregulated genes in the "PITPNC lo" group. (Data not shown) These genes were used to perform GO and KEGG enrichment analysis. Results of GO analysis showed that these genes were enriched in metabolic-related pathways that took part in ROS generation (Figure 2F). RNA-seq data was also used to perform GSEA, and 2 oxidative stress-related pathways showed significant enrichment in the "PITPNC1 lo" group, suggesting that rectal cancer tissue with low PITPNC1 
expression level may be under a condition of more intense oxidative stress (Figure 2G). Conclusively, these results suggest that high expression of PITPNC1 in rectal cancer is related to decreased ROS production.

\section{Maintenance of radioresistance in rectal cancer is PITPNC1-dependent}

Base on the relevance between radioresistance and high expression of PITPNC1, we next explored whether the maintenance of radioresistance was PITPNC1-dependent. ShPITPNC1 was used to knockdown PITPNC1 in HCT116 and SW620. To know whether knocking down PITPNC1 reversed radioresistance, flow cytometry was employed to detect the apoptosis rate of differently treated groups. Results showed that the application of shPITPNC1 elevated significantly the apoptosis rate in both irradiated and non-irradiated groups (Figure $3 A, B$ ). We next evaluated the effect of knocking down PITPNC1 on proliferation using colony formation assay. Similarly, we found that shPITPNC1 inhibited proliferation in both irradiated and non-irradiated groups (Figure 3C,D).

To further validate our findings, we performed western blotting to detect the level of phosphorylated H2A histone family member $\mathrm{X}$ on serine $139(\gamma \mathrm{H} 2 \mathrm{AX})$, the DNA double-strand break marker (31), and the apoptosis marker, cleaved Caspase-3 (32). Results showed that irradiation could increase the expression of both proteins, and the application of shPITPNC1 further intensified this effect (Figure 3E,F,G,H), suggesting that knocking down PITPNC1 sensitizes rectal cancer cell lines to irradiation damage. In conclusion, radioresistance in rectal cancer is dependent on PITPNC1.

\section{PITPNC1-dependent radioresistance in rectal cancer is mediated by inbibiting ROS generation}

As mentioned above, the rectal cancer cell line with the higher PITPNC1 expression, HCT116, showed less ROS generation than SW620 under both irradiated and nonirradiated conditions (Figure 2D,E). We thus explored whether this difference in ROS generation was resulted by the different expression levels of PITPNC1. We performed flow cytometry to analyze the effect of shPITPNC1 on ROS generation. A significantly higher ROS generation was observed in shPITPNC1 applied groups (Figure 4A,B). We then investigated whether the difference in radioresistance induced by different levels of PITPNC1 expression was
ROS generation mediated. Flow cytometry assay showed that apoptosis induced by shPITPNC1 was reversed by NAC, a potent ROS scavenger (Figure $4 C, D$ ). Similarly, colony formation assay showed that proliferation inhibited by knocking down PITPNC1 was reversed by applying NAC (Figure 4E,F). The apoptosis marker, cleaved Caspase-3 elevated by knocking down PITPNC1, was also partially reversed by NAC (Figure 4G,H,I,7). Of note, administrating NAC solely in shPITPNC1-free groups affected neither apoptosis nor proliferation (Figure 4C,D,E,F), suggesting the ROS generation-regulation effect of PITPNC1 strongly. Taking together, we demonstrated that rectal cancer radioresistance is mediated by PITPNC1-regulated inhibition of ROS generation.

\section{Discussion}

Neoadjuvant radiotherapy has become a crucial part of the comprehensive management of patients with rectal cancer in recent years, and its usage has significantly improved life quality and survival (4-6). Nevertheless, radioresistance remains a major challenge for further improvement of therapeutic effects of neoadjuvant radiotherapy and clinical decision-making. Also, there is a lack of a thorough understanding of the underlying mechanisms in the development of radioresistance is thus of great clinical importance.

Radiation-induced cell death initiates from the mass generation of ROS, which subsequently causes DNA doublestrand break and, consequently, apoptosis or necrosis (33). Previous studies have already identified multiple different possibilities to speculate the causes of the development of radioresistance in rectal cancer, including DNA repair system (34-39), apoptosis and cell survival (36,38-41), epithelialmesenchymal transition (EMT) $(37,42)$, tumor stem cell (43), and the generation of ROS (44), suggesting that the process of radioresistance-development may be complex.

For DNA repair system-related mechanisms, Ha Thi et al. reported that miR-130a targets sex determining region Y-box 4 (SOX4) and thus activates downstream ataxia-telangiectasia mutated (ATM)-mediated DNA repair (37). Rab5 and replication factor $\mathrm{C}$ subunit 4 (RFC4) are demonstrated to facilitate the expression of non-homologous end joining DNA repair-related proteins, $\mathrm{Ku} 70$ and $\mathrm{Ku} 80$ (34,35). In another recent study by Ferrandon et al., knocking down coenzyme A synthase (COASY) hindered DNA double-strand break (38). For apoptosis and cell surviving-related mechanisms, Survivin was found to play an important role in inhibiting apoptosis 
A

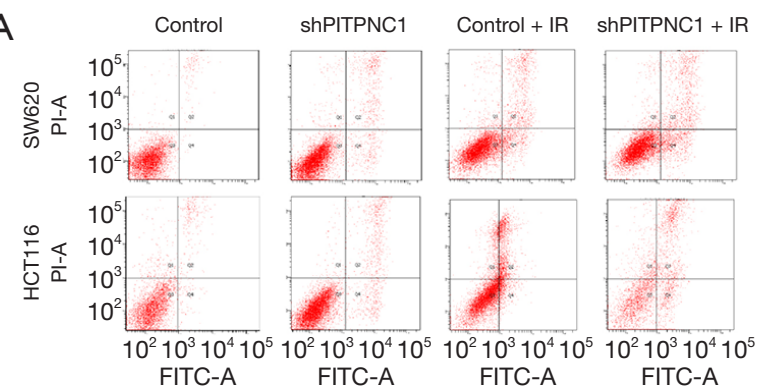

C

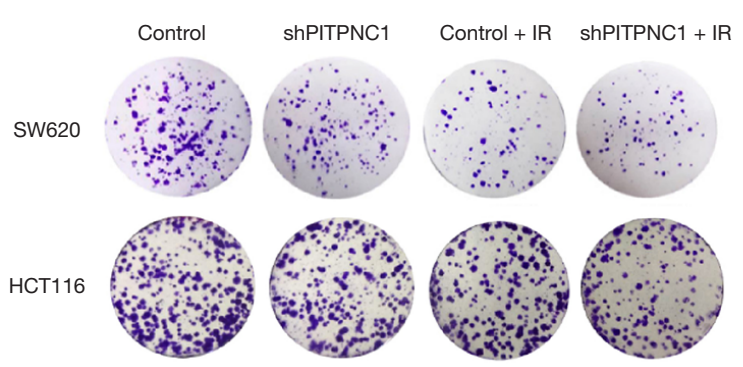

E

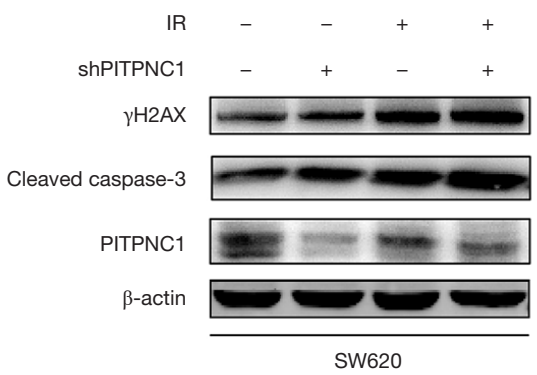

G

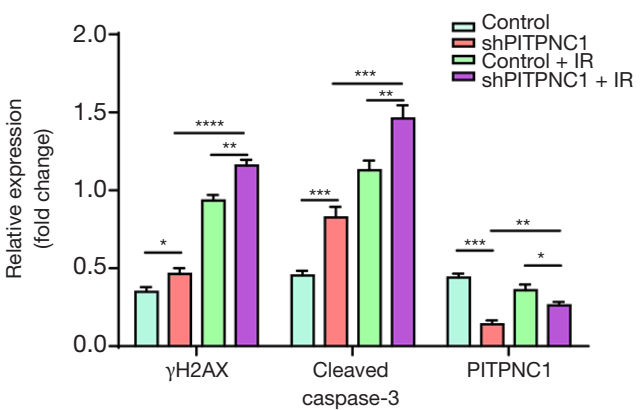

B
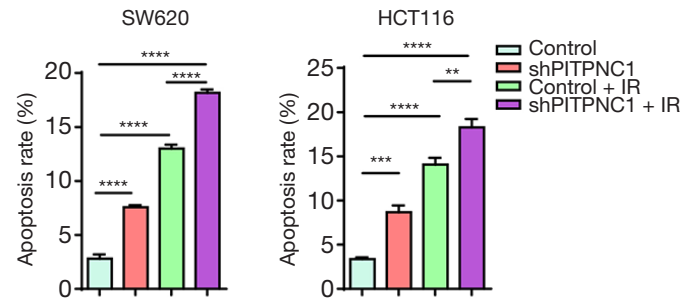

D

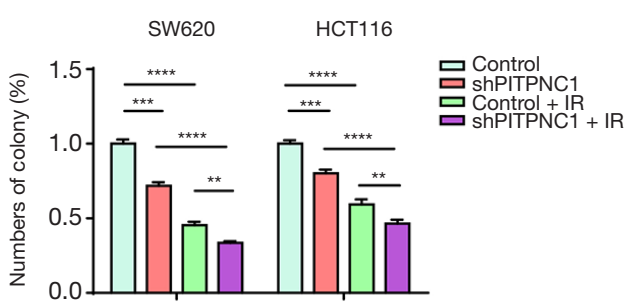

$\mathrm{F}$

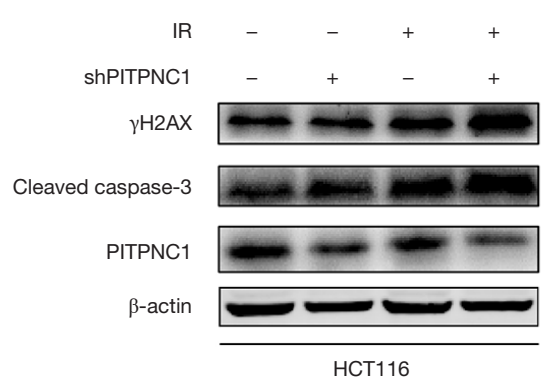

$\mathrm{H}$

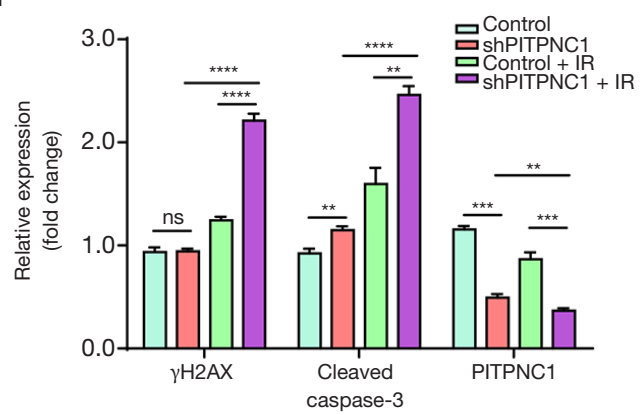

Figure 3 Knocking down of PITPNC1 reverses radioresistance. (A) Flow cytometry assessing the influence of knocking down PITPNC1 on apoptosis. Cultured SW620 and HCT116 cells were treated with irradiation exposure and/or shPITPNC1 transfection, with non-treated groups as a negative control; (B) quantization of flow cytometry results using an absolute apoptosis rate; (C) colony formation assay assessing the influence of knocking down PITPNC1 on proliferation. SW620 and HCT116 cells were treated with irradiation exposure and/or shPITPNC1 transfection. Non-treated groups served as a negative control. Cells were stained with crystal violet after fixation; (D) quantization of colony formation assay results normalized using the number of colonies in the control group. Western blot results of the level of DNA damage- and apoptosis-related proteins in the differently treated (E) SW620 and (F) HCT116 cells. And quantization of western blotting results of (G) SW620 and (H) HCT116 cells using relative expression level of $\gamma \mathrm{H} 2 \mathrm{AX}$, cleaved Caspase- 3 and PITPNC1 compared to $\beta$-actin. IR, irradiation; PITPNC1, phosphatidylinositol transfer protein, cytoplasmic $1 .{ }^{*}, \mathrm{P}<0.05 ;{ }^{* *}, \mathrm{P}<0.01$; ***, $\mathrm{P}<0.001$; ****, $\mathrm{P}<0.0001$. 
A

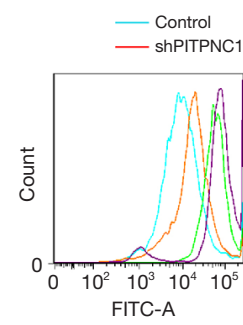

- Control + IR

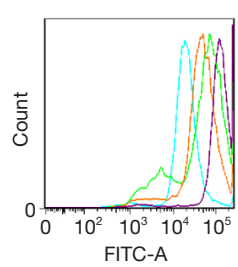

C
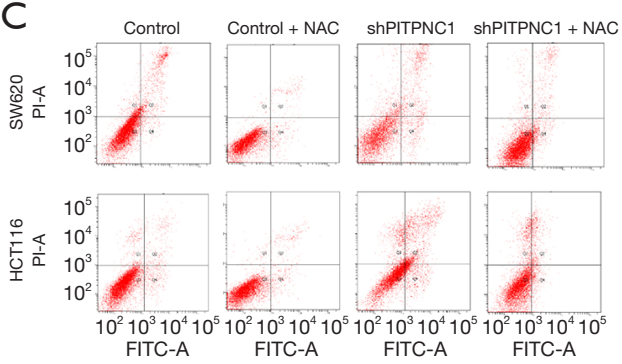

$E$

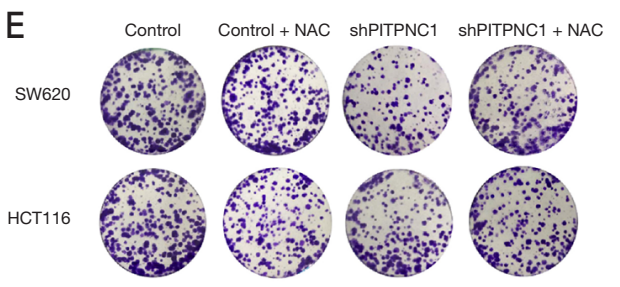

G

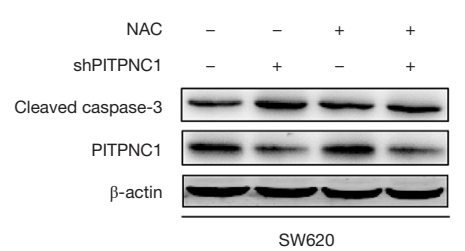

I

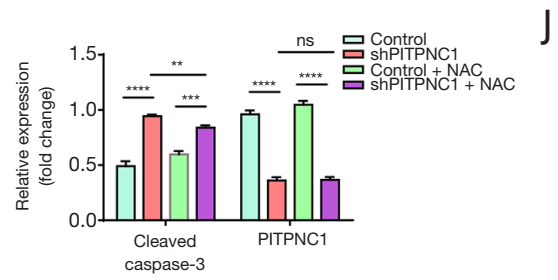

B

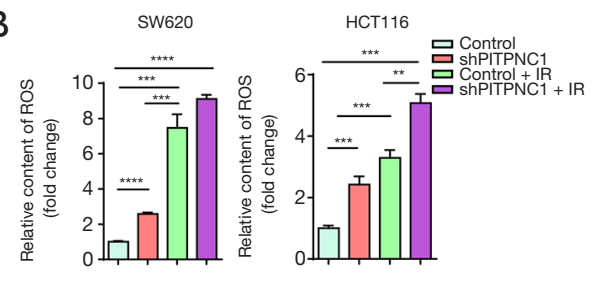

D
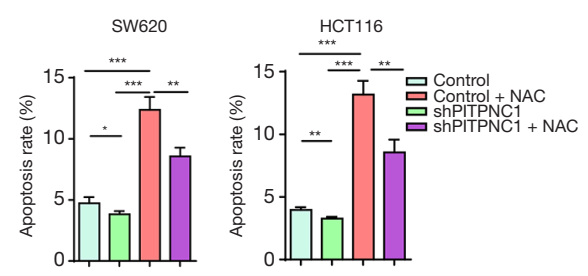

$\mathrm{F}$

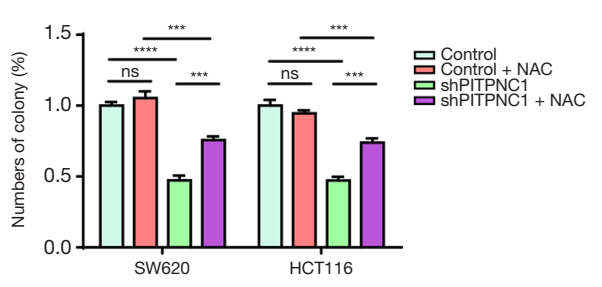

$\mathrm{H}$

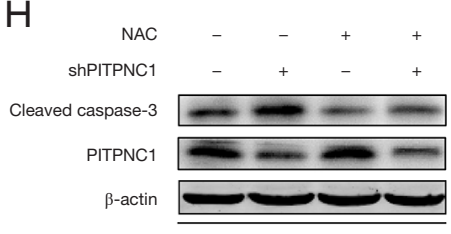

HCT116

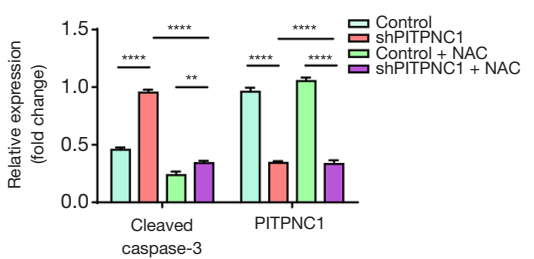

Figure 4 Knocking down of PITPNC1 reverses radioresistance via facilitating ROS generation. (A) Flow cytometry measuring the ROS generation under different treatments in SW620 (left) and HCT116 (right). SW620 and HCT116 cells were treated with irradiation exposure and/or shPITPNC1 transfection. Non-treated groups served as control; (B) quantization of flow cytometry results in panel (A) normalized using the level of the control group; (C) flow cytometry assessing the influence of ROS scavenger, NAC, on apoptosis. ShPITPNC1-transfected or -non-transfected SW620 and HCT116 cells were cultured in normal medium or medium with $2.5 \mathrm{nM} \mathrm{NAC;}$ (D) quantization of flow cytometry results in panel (C) using an absolute apoptosis rate; (E) colony formation assay assessing the influence of NAC on proliferation. ShPITPNC1-transfected or -non-transfected SW620 and HCT116 cells were cultured in normal medium or medium with 2.5 nM NAC. Cells were stained with crystal violet after fixation; $(\mathrm{F})$ quantization of colony formation assay results normalized using the number of colonies in the control group. Western blot data showing an assessment of the impacts of NAC on the cleavage of Caspase-3 protein in (G) SW620 and (H) HCT116 cell lines with $\beta$-actin serving as the loading control. And quantization of western blotting results of (I) SW620 and (J) HCT116 cells using relative expression level of cleaved Caspase- 3 and PITPNC1 compared to $\beta$-actin. IR, irradiation; PITPNC1, phosphatidylinositol transfer protein, cytoplasmic 1 ; ROS, reactive oxygen species; NAC, N-acetyl-L-cysteine. ${ }^{*}, \mathrm{P}<0.05$; **, $\mathrm{P}<0.01$; ${ }^{* * *}, \mathrm{P}<0.001$; ****, $\mathrm{P}<0.0001$. 
in rectal cancer $(39,41)$, whose expression was reported to be elevated by fibroblast growth factor 8 (FGF8) (36). Besides, as reported by Xiao et al., inhibiting retinoblastomabinding protein 6 (RBBP6) led to radiation-induced G2-M arrest and apoptosis via attenuation of $\mathrm{p} 53 /$ mouse double minute 2 (MDM2) interaction in rectal cancer (40). However, as the upstream initiator of these effects, very few studies investigated the role played by increased generation of ROS in rectal cancer radioresistance. Only one study by $\mathrm{Yu}$ et al. reported that multidrug resistance-associated protein 3 (MRP3) aggravates radioresistance by reducing ROS production (44). In this study, we for the first time demonstrated that the lipid metabolism-related protein, PITPNC1, fuels rectal cancer radioresistance via inhibition of the generation of ROS, consistent to a previous work which reported an increased mortality in rectal cancer patients carrying the low ROS producing endothelial nitric oxide synthase (eNOS) Glu298Asp asparagine allele compared to the homozygous carriers of the glutamine allele (45). Our findings add new information to the profile of the mechanisms in rectal cancer radioresistance.

In conclusion, we found that PITPNC1 was highly expressed in radioresistant patient-derived rectal cancer tissue compared to radiosensitive tissue; PITPNC1 inhibited the generation of ROS and improved the extent of radioresistance of rectal cancer cell lines and subsequently inhibited apoptosis. Knocking down PITPNC1 facilitated the production of ROS while application of the ROS scavenger, NAC, reversed this effect. Thus, our study proved that PITPNC1 fuels radioresistance of rectal cancer by inhibiting ROS production.

\section{Acknowledgments}

None.

\section{Footnote}

Conflicts of Interest: The authors have no conflicts of interest to declare.

Ethical Statement: The authors are accountable for all aspects of the work in ensuring that questions related to the accuracy or integrity of any part of the work are appropriately investigated and resolved. This study was approved by the Zhujiang Hospital (Guangzhou, China) Ethics Review Board (No. 2019-ZZ-003-03).

\section{References}

1. Siegel RL, Miller KD, Jemal A. Cancer statistics, 2019. CA Cancer J Clin 2019;69:7-34.

2. Kong L, Peng J, Li J, et al. Prolonged surveillance of colorectal cancer patients after curative surgeries beyond five years of follow-up. Ann Transl Med 2019;7:608.

3. Martin ST, Heneghan HM, Winter DC. Systematic review and meta-Analysis of outcomes following pathological complete response to neoadjuvant chemoradiotherapy for rectal cancer. Br J Surg 2012;99:918-28.

4. Ludmir EB, Palta M, Willett CG, et al. Total neoadjuvant therapy for rectal cancer: An emerging option. Cancer 2017;123:1497-506.

5. Clifford R, Govindarajah N, Parsons JL, et al. Systematic review of treatment intensification using novel agents for chemoradiotherapy in rectal cancer. Br J Surg 2018;105:1553-72.

6. Garcia-Aguilar J, Chow OS, Smith DD, et al. Effect of adding mFOLFOX6 after neoadjuvant chemoradiation in locally advanced rectal cancer: A multicentre, phase 2 trial. Lancet Oncol 2015;16:957-66.

7. Yu D, Wang Y, Song Y, et al. The efficacy of postoperative radiotherapy for locally advanced rectal cancer without neoadjuvant therapy. Transl Cancer Res 2018;7:922-35.

8. Cebrián A, Gómez del Pulgar T, Fernández-Aceñero MJ, et al. Decreased PLK1 expression denotes therapy resistance and unfavourable disease-free survival in rectal cancer patients receiving neoadjuvant chemoradiotherapy. Pathol Res Pract 2016;212:1133-7.

9. Voboril R, Rychterova V, Voborilova J, et al. NF- $\kappa B / p 65$ expression before and after treatment in rectal cancer patients undergoing neoadjuvant (chemo)radiotherapy and surgery: Prognostic marker for disease progression and survival. Neoplasma 2016;63:462-70.

10. Agostini M, Zangrando A, Pastrello C, et al. A functional biological network centered on XRCC3: A new possible marker of chemoradiotherapy resistance in rectal cancer patients. Cancer Biol Ther 2015;16:1160-71.

11. Wang HC, Chou CL, Yang CC, et al. Over-expression of CHD4 is an independent biomarker of poor prognosis in patients with rectal cancers receiving concurrent chemoradiotherapy. Int J Mol Sci 2019;20:4087.

12. Huang EY, Chang JC, Chen HH, et al. Carcinoembryonic antigen as a marker of radioresistance in colorectal cancer: A potential role of macrophages. BMC Cancer 2018; $18: 321$. 
13. Larosa $\mathrm{V}$, Remacle $\mathrm{C}$. Insights into the respiratory chain and oxidative stress. Biosci Rep 2018;38:1-14.

14. Bhatt AN, Chauhan A, Khanna S, et al. Transient elevation of glycolysis confers radio-resistance by facilitating DNA repair in cells. BMC Cancer 2015;15:335.

15. Yu L, Sun Y, Li J, et al. Silencing the Girdin gene enhances radio-sensitivity of hepatocellular carcinoma via suppression of glycolytic metabolism. J. Exp. Clin Cancer Res 2017;36:110.

16. Bol V, Bol A, Bouzin C, et al. Reprogramming of tumor metabolism by targeting mitochondria improves tumor response to irradiation. Acta Oncol 2015;54:266-74.

17. Tang L, Wei F, Wu Y, et al. Role of metabolism in cancer cell radioresistance and radiosensitization methods. J Exp Clin Cancer Res 2018;37:87.

18. Tan Z, Xiao L, Tang M, et al. Targeting CPT1A-mediated fatty acid oxidation sensitizes nasopharyngeal carcinoma to radiation therapy. Theranostics 2018;8:2329-47.

19. Kim W, Son B, Lee S, et al. Targeting the enzymes involved in arachidonic acid metabolism to improve radiotherapy. Cancer Metastasis Rev 2018;37:213-25.

20. Cockcroft S, Garner K. Function of the phosphatidylinositol transfer protein gene family: is phosphatidylinositol transfer the mechanism of action? Crit. Rev. Biochem. Mol Biol 2011;46:89-117.

21. Cockcroft S, Garner K. Potential role for phosphatidylinositol transfer protein (PITP) family in lipid transfer during phospholipase C signalling. Adv Biol Regul 2013;53:280-91.

22. Epand RM. Features of the Phosphatidylinositol Cycle and its Role in Signal Transduction. J Membr Biol 2017;250:353-66.

23. Grabon A, Orłowski A, Tripathi A, et al. Dynamics and energetics of the mammalian phosphatidylinositol transfer protein phospholipid exchange cycle. J Biol Chem 2017;292:14438-55.

24. Grabon A, Bankaitis VA, McDermott MI. The interface between phosphatidylinositol transfer protein function and phosphoinositide signaling in higher eukaryotes. J Lipid Res 2019;60:242-68.

25. Garner K, Hunt AN, Koster G, et al. Phosphatidylinositol transfer protein, cytoplasmic 1 (PITPNC1) binds and transfers phosphatidic acid. J Biol Chem 2012;287:32263-76.

26. Png KJ, Halberg N, Yoshida M, et al. A microRNA regulon that mediates endothelial recruitment and metastasis by cancer cells. Nature 2011;481:194-6.

27. Halberg N, Sengelaub CA, Navrazhina K, et al. PITPNC1
Recruits RAB1B to the Golgi Network to Drive Malignant Secretion. Cancer Cell 2016;29:339-53.

28. Tan Y, Lin K, Zhao Y, et al. Adipocytes fuel gastric cancer omental metastasis via PITPNC1-mediated fatty acid metabolic reprogramming. Theranostics 2018;8:5452-68.

29. Mootha VK, Lindgren CM, Eriksson KF, et al. PGC-1 $\alpha$ responsive genes involved in oxidative phosphorylation are coordinately downregulated in human diabetes. Nat Genet 2003;34:267-73.

30. Subramanian A, Tamayo P, Mootha VK, et al. Gene set enrichment analysis: A knowledge-based approach for interpreting genome-wide expression profiles. Proc Natl Acad Sci U S A 2005;102:15545-50.

31. Siddiqui MS, François M, Fenech MF, et al. Persistent $\gamma \mathrm{H} 2 \mathrm{AX}$ : A promising molecular marker of DNA damage and aging. Mutat Res Rev Mutat Res 2015;766:1-19.

32. Shang N, Bank T, Ding X, et al. Caspase-3 suppresses diethylnitrosamine-induced hepatocyte death, compensatory proliferation and hepatocarcinogenesis through inhibiting p38 activation. Cell Death Dis 2018;9:558.

33. Herst PM, Grasso C, Berridge M V. Metabolic reprogramming of mitochondrial respiration in metastatic cancer. Cancer Metastasis Rev 2018;37:643-53.

34. Baptistella AR, Landemberger MC, Dias MVS, et al. Rab5C enhances resistance to ionizing radiation in rectal cancer. J Mol Med 2019;97:855-69.

35. Wang XC, Yue X, Zhang RX, et al. Genome-wide RNAi Screening Identifies RFC4 as a Factor That Mediates Radioresistance in Colorectal Cancer by Facilitating Nonhomologous End Joining Repair. Clin Cancer Res 2019;25:4567-79.

36. Harpain F, Ahmed MA, Hudec X, et al. FGF8 induces therapy resistance in neoadjuvantly radiated rectal cancer. J Cancer Res Clin Oncol 2019;145:77-86.

37. Ha Thi HT, Kim HY, Kim YM, et al. MicroRNA-130a modulates a radiosensitivity of rectal cancer by targeting SOX4. Neoplasia 2019;21:882-92.

38. Ferrandon S, DeVecchio J, Duraes L, et al. CoA Synthase (COASY) Mediates Radiation Resistance via PI3K Signaling in Rectal Cancer. Cancer Res 2020;80:334-46.

39. Rödel F, Hoffmann J, Distel L, et al. Survivin as a radioresistance factor, and prognostic and therapeutic target for radiotherapy in rectal cancer. Cancer Res 2005;65:4881-7.

40. Xiao C, Wang Y, Zheng M, et al. RBBP6 increases radioresistance and serves as a therapeutic target for preoperative radiotherapy in colorectal cancer. Cancer Sci 
2018;109:1075-87.

41. Rödel C, Haas J, Groth A, et al. Spontaneous and radiation-induced apoptosis in colorectal carcinoma cells with different intrinsic radiosensitivities: Survivin as a radioresistance factor. Int J Radiat Oncol Biol Phys 2003;55:1341-7.

42. Shao M, Bi T, Ding W, et al. OCT4 Potentiates RadioResistance and Migration Activity of Rectal Cancer Cells by Improving Epithelial-Mesenchymal Transition in a ZEB1 Dependent Manner. Biomed Res Int 2018;2018:3424956.

43. Ji D, Zhan T, Li M, et al. Enhancement of Sensitivity to Chemo/Radiation Therapy by Using miR-15b against

Cite this article as: Tan Y, Shao R, Li J, Huang H, Wang Y, Zhang M, Cao J, Zhang J, Bu J. PITPNC1 fuels radioresistance of rectal cancer by inhibiting reactive oxygen species production. Ann Transl Med 2020;8(4):126. doi: 10.21037/ atm.2020.02.37
DCLK1 in Colorectal Cancer. Stem Cell Reports 2018;11:1506-22.

44. Yu Z, Zhang C, Wang H, et al. Multidrug resistance-associated protein 3 confers resistance to chemoradiotherapy for rectal cancer by regulating reactive oxygen species and caspase-3-dependent apoptotic pathway. Cancer Lett 2014;353:182-93.

45. Funke S, Risch A, Nieters A, et al. Genetic Polymorphisms in Genes Related to Oxidative Stress (GSTP1, GSTM1, GSTT1, CAT, MnSOD, MPO, eNOS) and Survival of Rectal Cancer Patients after Radiotherapy. J Cancer Epidemiol 2009;2009:302047. 